\title{
Langmuir and LB Properties of Two Calix[4]resorcinarenes: Interactions With Various Analytes
}

\author{
Mark W. Sugden ${ }^{a}$, Tim H. Richardson ${ }^{a, *}$, F. Davis ${ }^{b}$, Seamus P.J. Higson ${ }^{b}$ and Charl F.J. Faul ${ }^{c}$ \\ ${ }^{a}$ Department of Physics and Astronomy, University of Sheffield, Hounsfield Road, Sheffield S3 7RH \\ ${ }^{b}$ Cranfield Biotechnolgy Centre, Cranfield University, Silsoe Campus, Cranfield \\ ${ }^{c}$ School of Chemistry, Inorganic and Materials Chemistry, University of Bristol, Bristol, BS8 1 TS \\ ${ }^{*}$ Corresponding author E-mail address: m.sugden@sheffield.ac.uk
}

\begin{abstract}
The ability of calixarene based molecules to interact with amino acids has been the basis of many studies. The Langmuir and LB properties of two calix[4]resorcinarenes have been investigated. The properties of the layer formed at the air-water interface were studied by surface pressure area isotherms. LB deposition onto glass substrates has shown that multilayer assemblies can be built up. The UV-visible spectra of resulting LB films have been recorded, indicating that the compounds are coloured as a result of intra-molecular charge transfer bands. The sensitivity of the surface pressure area isotherms has been investigated in relation to the exposure to various analytes delivered from the subphase (i.e. amino acids). Furthermore, exposure of the LB films to a wide range of vapours (e.g. amines, alcohols, thiols) has led to modified UV-visible spectra.
\end{abstract}

\section{Introduction}

Calix[4] resorcinarene derivatives in the form of thin films are of particular interest within the sensing community due to their selective recognition of different molecules. Films of controlled thickness of these calix[4]resorcinarenes can be created using the Langmuir-Blodgett technique[1]. Different calixresorcinarene derivatives have been deposited onto varying substrates as thin films by the LB technique which were then used as chemical sensors[2]. The cavity of the resorcinarene provides a site for the binding of organic guest molecular species [3] and the selectivity of the resorcinarene to particular analytes can be controlled somewhat by altering the size of the cavity as well as the peripheral substituted groups. Most commonly, the cavity size corresponds to $\mathrm{n}=4,6$ or 8 . A wide range of analyte species has been previously studied including metal ions [4] and amino acids [5]. Various analytical techniques have been used to follow the sensing response including UV-visible spectroscopy [6], surface plasmon resonance [7] and quartz crystal resonance [8].

\section{Materials}

In this work, two calix[4]resorcinarenes have been studied in solution, Langmuir film and LB film form. The chemical structures of these materials, Calix 1 and Calix 2, are shown in Figure 1. The materials were first dissolved in chloroform, and then for isotherm and LB film studies they were spread over the water surface to form floating Langmuir films. These materials used are both coloured and it is thought that this colour is the result of intramolecular charge transfer bands.

Both these materials are amphiphilic and so are expected to form well ordered Langmuir films. A wide range of acids, amines and thiols used as analytes have been exposed to the calix[4]resorcinarenes. The analytes used were acetic acid, ethyl acetate, hexanethiol, hexylamine, octanol, octanal, octylamine, triethylamine, trimethylphosphate and three amino acids. The amino acids used were lysine, alanine and glutamic acid. These were chosen because of their acidic or basic nature, lysine being basic, glutamic acid being acidic and alanine being neutral. By investigating such a large number of analytes, the degree of selectivity of 


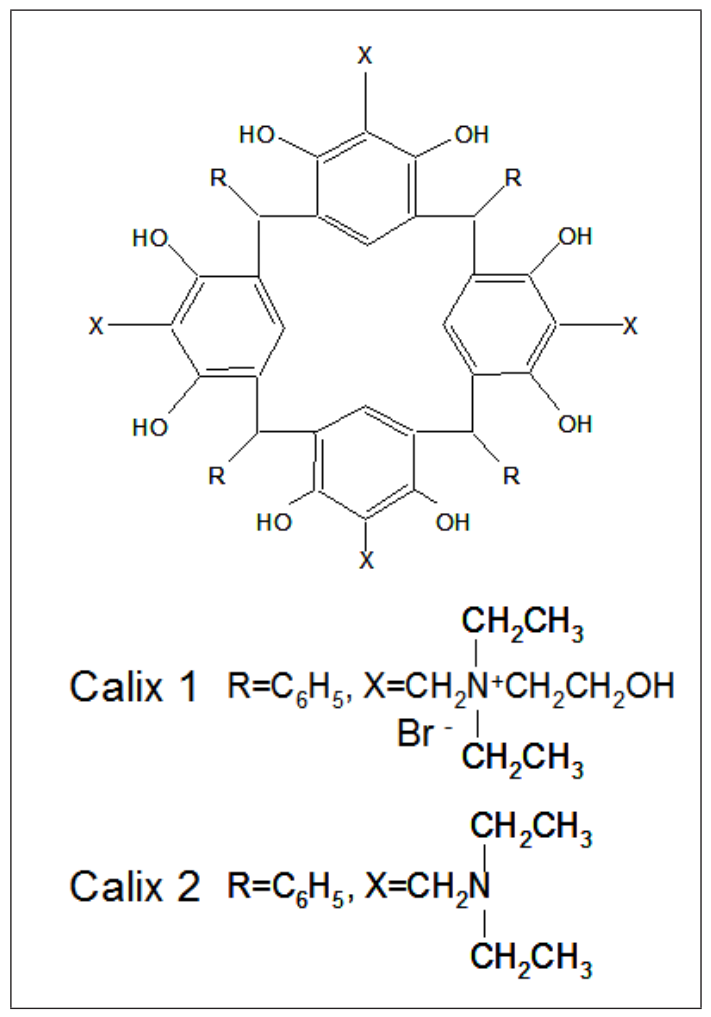

Fig. 1. Chemical structure of the calix[4]resorcinarenes

each calix[4]resorcinarene can be established.

\section{Experimental Details}

\subsection{LB film production}

Chloroform solutions of the calix[4]resorcinarenes were produced, the concentrations used were 0.292 $\mathrm{g} / \mathrm{l}$ and $1.093 \mathrm{~g} / \mathrm{l}$ for Calix 1 and 2 respectively. A volume of $50 \mu \mathrm{l}$ of the solution was spread, using a syringe, onto the surface of the water subphase $(>15 \mathrm{M} \Omega \mathrm{cm})$ within a NIMA Langmuir-Blodgett trough (Type 611). A period of 10 minutes was given to allow any excess chloroform to evaporate off the surface before the compression was initiated. The speed of the compression was set to $40 \mathrm{~cm}^{2} \mathrm{~min}^{-1}$.

Using the LB technique 20 layers of each of the materials were transferred onto a clean, hydrophobic glass substrate. The surface pressure of the material was held constant at $15 \mathrm{mN} / \mathrm{m}$ and when this pressure was attained the deposition process was initiated at a speed of $5 \mathrm{~mm} / \mathrm{min}$.

For the investigation into the calix[4] resorcinarene interaction with the amino acids, the amino acids were first dissolved in water. The amino acids were added to the subphase after the material had been spread and the 10 minutes evaporation time had elapsed. The amino acids were introduced to the subphase outside of the area confined by the barriers, in order not to disturb the floating layer. The trough used had a volume of $350 \mathrm{ml}$ and the amount of amino acid solution placed into the subphase was sufficient to produce a concentration of 0.01 $\mathrm{M}$ for each material.

\subsection{Spectroscopy}

UV-visible spectra of the materials were recorded after they had been deposited onto glass substrates. These spectra were obtained using the Spectromate micro spectrophotometer (World Precision Instruments). When the spectra had been recorded, the substrate was then immersed in a $0.01 \mathrm{M}$ solution of each amino acid for 30 minutes. Following this process, the spectra of the samples were monitored again.

Solution spectroscopy of these two materials was also performed using the Ocean Optics UV-Vis lightsource (Model: Mini-D2) and spectrometer (USB2000) with a wavelength range 179nm $876 \mathrm{~nm}$. The concentrations used for this process were $0.052 \mathrm{~g} / \mathrm{l}$ and $0.055 \mathrm{~g} / \mathrm{l}$ for calix 1 and 2 respectively. A quartz cuvette was used and $850 \mu$ l of the soultions were placed within it. The sectrum was first taken and then the solution was mixed $100 \mu \mathrm{l}$ of each nine different analytes and then spectra readings were repeated.

\section{Results and discussion}

\subsection{Surface Pressure-Area Isotherms}

Isotherms of both Calix 1 and 2 were recorded on a Langmuir trough (Type 601BAM). The expected approximate area per molecule for the calix[4]resorcinare unit is in the range 1.2 $1.3 \mathrm{~nm}^{2}$. Therefore, the extrapolated area per molecule measured for Calix $1\left(1.1 \mathrm{~nm}^{2}\right)$ suggests that a monolayer is formed at the air-water interface. The corresponding value for Calix 2 is $0.75 \mathrm{~nm}^{2}$, indicating most probably that this calix[4]resorcinarene is aggregating in the spreading solution to a greater extent than that of Calix 1 .

After the subphase had been doped with amino acids, several of the isotherms showed differences from their original form. The results of each of the isotherms from the three amino acids can be seen in Figure 2. 


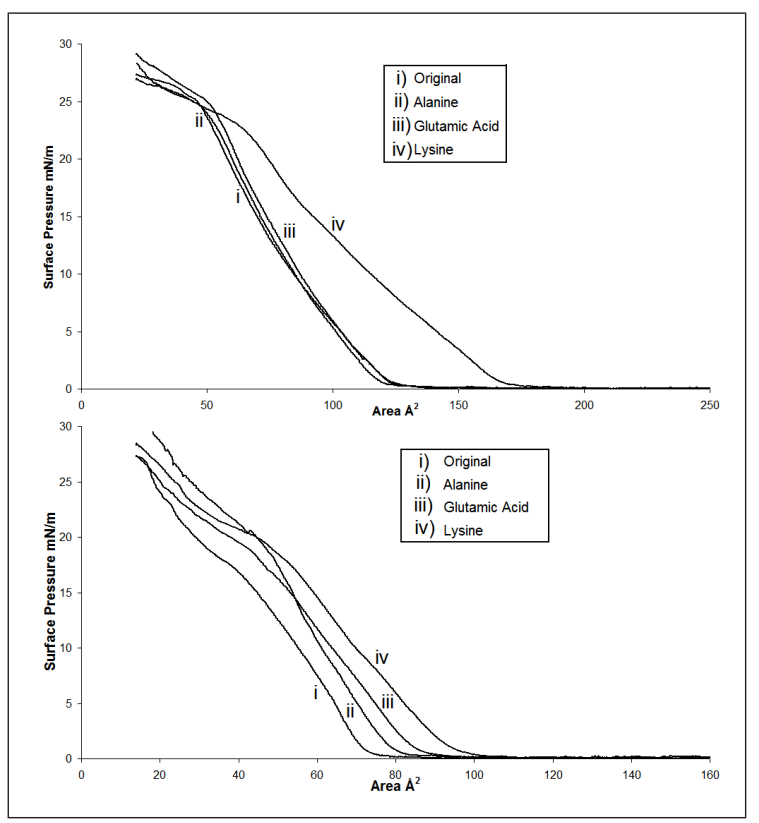

Fig. 2. Isotherms before and after doping the water subphase with various amino acids, showing their effects on a) Calix 1 and b) Calix 2

While studying Calix 1, the suphase was first doped with alanine. However with this amino acid there was no noticeable change to the isotherm curve. Similarly when the subphase was doped with glutamic acid no change in the isotherm curve was present. When lysine was added to the subphase, there was a distinct modification to the isotherm. The intial increase in surface pressure, the phase change from the gaseous to the solid phase, occurred for a much higher area per molecule. This change showed that Calix 1 had interacted with lysine on the water surface and had caused an apparent expansion of the film on the surface.

Calix 2 did not follow the same trend as Calix 1, showing interactions with each of the three amino acids. The area per molecule at the point where the surface pressure rises above $0 \mathrm{mN} / \mathrm{m}$ for each of the three amino acids. However the changes were less significant than for Calix 1. The greatest change was again caused by the doping the water with lysine and alanine produced the least reaction on the water surface.

\subsection{Solution Spectroscopy}

Both of the calix[4]resorcinarenes under discussion were then investigated to determine their possible use as sensors for the range of analytes mentioned in section 2 . This was initially carried out with the materials in a solution of chloroform. Spectra of Calix 1 and 2 were taken in solution before and after their exposure to these analytes. Examples

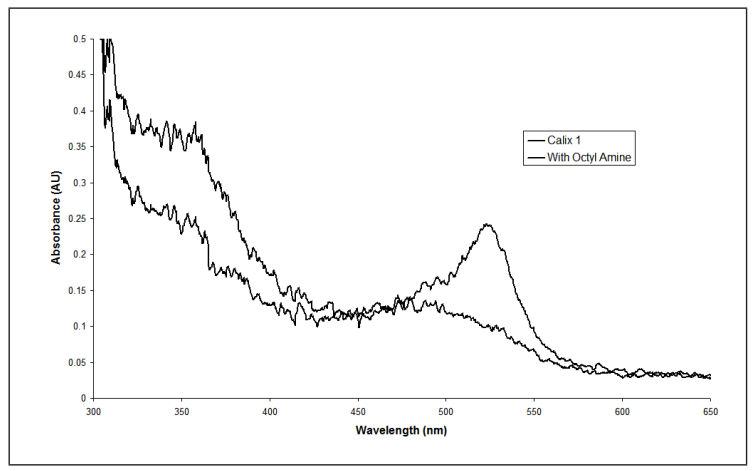

Fig. 3. Absorbance spectra of Calix 1 solution before and after the addition of octylamine

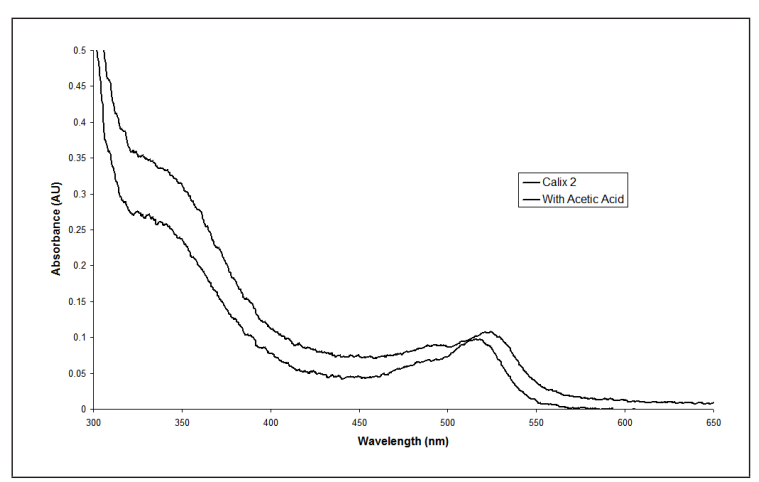

Fig. 4. Absorbance spectra of Calix 2 solution before and after the addition of acetic acid

of the changes caused by introducing the analyte which caused the largest change in the spectra of calix 1 and 2 are shown in figure 3 and figure 4 respectively. The reduction in the adsorption intensity around $525 \mathrm{~nm}$ in figure 3 is thought to occur as a result of the adsorption of amine which may hinder the intra-molecular charge transfer process.

The resulting absorbance changes, between $350 \mathrm{~nm}$ - 650nm, for both Calix 1 and 2 for each of the analytes used can be seen in Figure 5 .

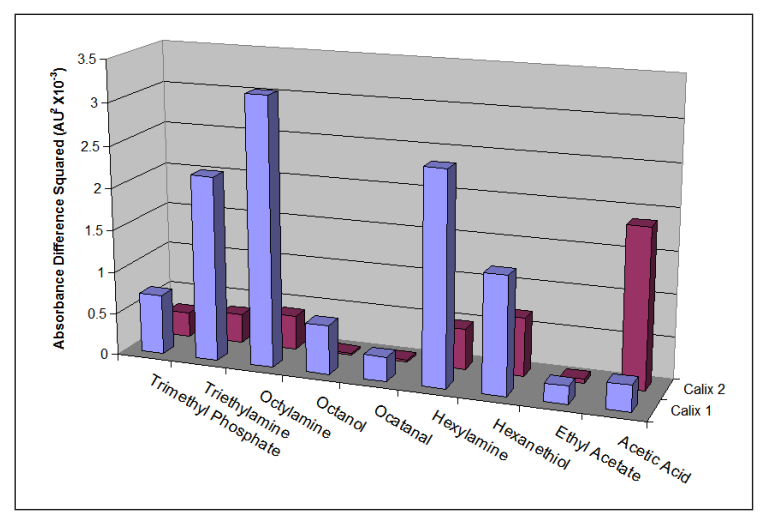

Fig. 5. Differences in the absorbance spectra squared of the solutions in the wavelength region between $350 \mathrm{~nm}-650 \mathrm{~nm}$

When studying Calix 1, three of the analytes used resulted in a distinct change in the spectra of the material; triethylamine, octylamine and hexylamine. Other analytes such as trimethylphos- 
phate and hexanethiol produced a lesser change in the spectra also. These effects show that Calix 1 is sensitive to the presence of amines. The sensitivity of Calix 1 to amines is due to the fact that this calix contains eight phenolic hydroxyl groups which have a pKa of approximately 9-10 [9][10]. However only four of these can be deprotonated, much more drastic conditions are required to remove any more protons. Addition of basic species such as amines causes formation of charged phenolic groups, thereby affecting the spectrum.

As our results show, Calix 2 is sensitive to acetic acid but is insensitive to the presence of amines whereas the opposite is true for Calix 1. Calix 2 also reacted, but with a much smaller response, when mixed with hexanethiol. Calix 2 contains both hydroxyl and amine groups. It is thought that within this system, self-protonation occurs where four of the hydroxyl groups protonate the amine groups [11]. Since this system is inherently already deprotonated, it follows that addition of further amines will only have minimal effects since they are not strong enough bases to remove any of the remaining four hydroxyl protons. However since acetic acid is more acidic than phenolic groups, addition of the acid reprotonates the hydroxyls, again leading to changes in the sectrum.

\subsection{LB film spectroscopy}

Samples were made from Calix 1 and 2 that consisted of 20 layers on a side of the glass substrate. After recording the initial spectra, the samples were then immersed in a solution of each amino acid, again with a concentration of $0.01 \mathrm{M}$. The spectra were then recorded again. The resulting changes in the spectra from this process can be seen in Figure 6.

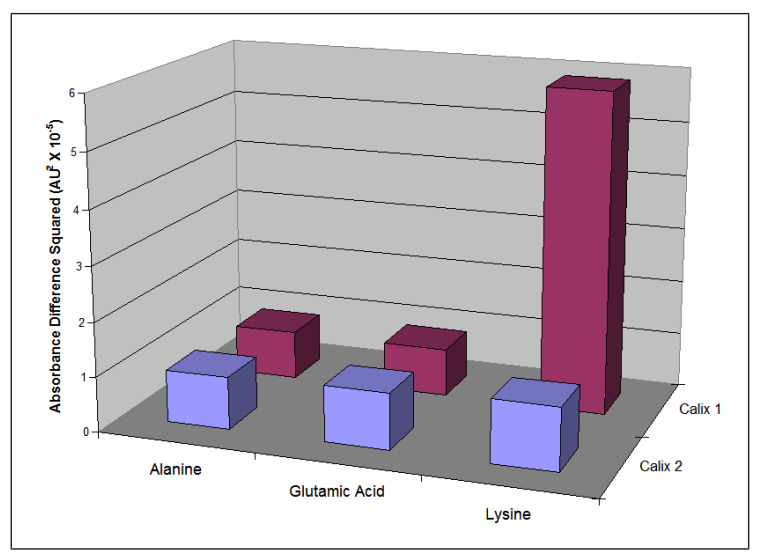

Fig. 6. Differences in the absorbance spectra squared of the $20 \mathrm{LB}$ layer samples in the wavelength region between $350 \mathrm{~nm}-650 \mathrm{~nm}$
The changes observed for Calix 1 are in agreement with the isotherm work previously discussed. Both alanine and glutamic acid produced almost no change in the spectra recorded. Lysine produced the greatest change in the observed spectra, which is in excellent agreement with the isotherm results.

The differences observed in the for Calix 2 spectra are also in agreement with the results from the isotherm work. These changes are much less pronounced in comparison to the changes produced with Calix 1.

\section{Conclusions}

The sensing behaviour of two calix[4]resorcinarenes has been characterised in terms of modifications to their surface pressure - area isotherms, their UV-visible solution and LB film spectra, for a wide range of analytes. In particular, LB films of Calix 1 has proved to bind selectively to lysine, an important amino acid. This is not the first time that strong interactions have been observed between calix-type molecules and amino acids. Selkti et al [12] described the binding of lysine by a sulfonatocalix[4] arene although this was not in LB film form. An attempt was made to investigate the interaction between the calix[4]resorcinarenes and the amino acids in solution, it was thought that ethanol would be suitable but the amino acids would not fully dissolve in the solvent. Further to this work studies will be carried out into the reactions occuring between LB films of both of the calix[4]resorcinarenes to a more extensive range of analytes including additional amino acids.

\section{Acknowledgements}

We would like to thank the EPRSC for financial support.

\section{References}

[1] A.V. Nabok, N.V. Lavrik, Z.I. Kazantseva, B.A. Nesterenko, L.N. Markovskiy, V.I. Kalchenko, and A.N. Shivaniuk. Complexing properties of calix[4]resorcinarene lb films. Thin Solid Films, 259:244-247, 1995.

[2] A.K. Hassan, A.K. Ray, A.V. Nabok, and F. Davis. Spun films of novel calix[4]resorcinarene derivatives for benzene vapour sensing. Sensors and Actuators B, 77:638-641, 2001. 
[3] M.M. Stone, A.H. Franz, and C.B. Lebrilla. Noncovalent calixarene-amino acid complexes formed by maldi-ms. J. Am. Soc. Mass Spectrom., 13:964-974, 2002.

[4] X. Hu, Z. Pan, L. Wang, and X. Shi. The molecular recognition of tetra(p-t-butyl)tetrathiocalix[4]arene and its derivatives to heacy metal ions. Spectrochim. Acta, Part A, 59:2419-2423, 2003.

[5] W.M. Hassen, C. Martelet, F. Davis, S.P.J. Higson, A. Abdelghani, and N. Jaffrezic-Renault. Calix[4] arene based molecules for amino-acid detection. Sensors and Actuators B: Chemical, 124:38-45, 2007.

[6] O. Omar, A.K. Ray, A.K. Hassan, and Davis F. Resorcinol calixarenes (resorcinarenes): Langmuirblodgett films and optical properties. Supramolecular Science, 4:417-421, 1997.

[7] A.K. Hassan, A.V. Nabok, A.K. Ray, A. Lucke, K. Smith, C.J.M. Stirling, and F. Davis. Thin films of calix-4-resorcinarene deposited by spin coating and Langmuir-Blodgett techniques: determination of film parameters by surface plasmon resonance. Mat.Sci.Eng.C, 8:251-255, 1999.

[8] X.C. Zhou, S.C. Ng, H.S.O. Chan, and S.F.Y. Li. Detection of organic amines in liquid with chemically coated quartz crystal microbalance devices. Sensors and Actuators B: Chemical, 42:137-144, 1997.

[9] F. Davis and C.J.M. Stirling. Calix-4-resorcinarene monolayers and multilayers: Formation, structure and differential adsorption. Langmuir, 12:5365-5374, 1996.

[10] E.K. Kazakova, N.A. Makarova, A.U. Ziganshina, L.A. Muslinkina,

Muslinkin, and W.D. Habicher. Novel water-soluble tetrasulfonatomethylcalix[4]resorcinarenes.

Tetrahedron Lett., 41:10111-10115, 2000.

[11] D.A. Leigh, P. Linnane, R.G. Pritchard, and G. Jackson. Unusual host-guest pi-arene ... h-bonding in a hooded cavitand - the first solid state structure of a calix[4]resorcinarene with underivatized hydroxy groups. Journal of the Chemical Society - Chemical Communications, 4:389-390, 1994.

[12] M. Selkti, A.W. Coleman, I. Nicolis, N. DouteauGuevel, F. Villain, A Tomas, and C. De Rango. The first example of a substrate spanning the calix[4]arene bilayer: the solid state complex of psulfonatocalix[4]arene with l-lysine. Chem. Comm., pages 161-162, 2000. 